\title{
PERCEPCIÓN SOBRE EL IMPACTO DEL TURISMO EN GUANACASTE, COSTA RICA
}

\section{PERCEPTION OF THE IMPACT OF TOURISM IN GUANACASTE, COSTA RICA}

\author{
Albert Espinoza-Sánchez ${ }^{1}$ \\ ${ }^{1}$ Costarricense. Docente e investigador. Universidad de Costa Rica. Sede Guanacaste. Email: \\ albert.espinoza@ucr.ac.cr
}

Recibido: 10.11 .18 Aprobado: 30.03 .19

DOI:10.15517/isucr.v20i41.38820

\section{Resumen}

Mucho se ha dicho sobre el impacto del Turismo en Guanacaste, unos indican que ha sido un motor de crecimiento en la provincia; otros, que sus beneficios no han salido del bolsillo de las operadoras de Turismo. Se han hecho estudio de impacto económico de la actividad turística, pero hasta ahora no se ha realizado un estudio que mida la percepción que tienen los guanacastecos respecto del impacto de esta actividad. Este es el primer estudio de enfoque cuantitativo, con una muestra representativa de 640 personas de toda la provincia, que da respuesta a la siguiente interrogante ¿qué opinión tienen los residentes en Guanacaste sobre el impacto del Turismo en sus comunidades? Además de dar respuesta a esa pregunta, se hacen comparaciones de esta opinión respecto de diferentes variables sociodemográficas, así como se destacan los beneficios y problemas atribuidos al Turismo.

Palabras claves: Turismo; percepción; Guanacaste; economía; Costa Rica;

\begin{abstract}
Much has been said about the impact of tourism in Guanacaste; while some consider it the engine which has helped the province to grow, others claim that its benefits are not the result of the work of tourism operators. Economic impact studies of tourism have been previously carried out; however, up until now no study has been able to measure the perception people from Guanacaste has about it. This is the first quantitative approach study, with a representative sample of 640 people from all over the province, which answers the following question: what is the opinion of residents in Guanacaste about the impact of tourism on their communities? This paper also compares this opinion according to different sociodemographic variables, and presents the benefits and problems attributed to tourism.

Key words: Tourism; perception; Guanacaste; economy; Costa Rica;
\end{abstract}




\section{Antecedentes}

Por mucho tiempo mucho se ha especulado sobre los beneficios y problemas derivados de la industria turística, unos exaltan los beneficios; otros, cuestionan que los beneficios han sido poco y no han calado en el desarrollo de la provincia. Sin embargo, hasta ahora, no se han realizado estudios de corte cuantitativo para medir la percepción que tienen los residentes en Guanacaste sobre esta actividad. Sí ha habido estudios sobre el impacto económico o estudios de corte cualitativo que señalan beneficios o problemas, pero solo en determinados lugares de la región; por lo tanto, no existen estudios referidos a la globalidad de la provincia. A continuación, se presentan investigaciones con referencia a este tema.

Arrieta y Rivera (2009, 141-154) relacionan el inicio del Turismo en la provincia con la creación de la Junta Nacional de Turismo en el año 1931; este organismo nació principalmente para promover esta actividad en el Valle Central. Aprovechando la existencia de esta organización, un grupo de guanacastecos que residían en dicha región, unidos en una organización denominada Casa de Guanacaste, se dan la tarea de impulsar la actividad turística en la provincia mediante la citada Junta. Eran tiempos en donde la actividad ganadera no estaba satisfaciendo las necesidades de empleo ni el crecimiento económico, por lo que se hacía necesaria otra actividad comercial que diera respiro a la alicaída economía guanacasteca, afectada por las consecuencias mundiales de la I Guerra Mundial y la depresión de 1929. Se concibe así, por primera vez, al Turismo como la panacea económica de la provincia.

Coinciden estos esfuerzos con el interés de los gobiernos de la época por mejorar los contactos con las provincias costeras. Hasta el nacimiento del ICT en 1955, se gestan campañas, principalmente en medios periodísticos, para llevar turistas a Guanacaste, promoviendo principalmente las playas y, entre estas, las Playas del Coco. En las décadas de los años 60 y 70 el turismo toma rasgo institucional y se promueve a nivel nacional. Pero es hasta la década de los años 80 cuando Guanacaste resurge como destino turístico enfocado en el desarrollo turístico en el Golfo de Papagayo, cuyo proyecto tiene origen en un estudio realizado por el Banco Centroamericano de Integración Económica, que identificó este lugar como el mejor en el Istmo para la construcción del complejo turístico actual.

Sin embargo, el gran cambio, apuntan estos autores, se da en los años 90 con investigaciones dirigidas a determinar las preferencias de los turistas, en donde cuales se determina que solo un poco más de la cuarta parte de los turistas que visitaban el país lo hacía en Guanacaste. Esto permitió desarrollar estrategias para incrementar esta actividad en la región.

En los últimos 20 años el turismo en Guanacaste ha experimentado un acelerado crecimiento principalmente en enfocado en la explotación hotelera en las playas, lo cual ha generado un alto impacto en la dimensión social, cultural y económica de la provincia. (Córdoba, Loría y Picón, 2011, pág. 193).

Castellón (2005, págs. 67-113) realizó un estudio de percepción (enfoque cualitativo) sobre el impacto del turismo en un grupo de pobladores de la zona costera de Santa Cruz, cerca de 
Playa Tamarindo. En la dimensión económica, este estudio encontró una percepción más positiva que negativa entre estos pobladores, destacando la actividad turìstica como generadora de ingresos económicos para las familias, principalmente vía salarios ya que son pocos los pobladores que tienen sus propias empresas. Pero, aun así, el turismo resulta atractivo como lugar de trabajo, por considerarse los ingresos obtenidos en esta actividad como mejores, comparados con los percibidos en otras actividades productivas de la región. Esto a pesar de los factores percibidos como negativos relacionados con el turismo, tales como la inestabilidad laboral, el trabajo estacional o por temporadas.

Destaca, además, que los pobladores perciben que antes se sembraba más y había trueque de productos entre vecinos, pero ahora la mayoría se dedica a laborar en Turismo porque consideran que les deja mejores ingresos económicos. En cuanto al desarrollo comunal, el autor encontró que estos pobladores perciben que no hay un significativo progreso ligado a esta actividad, sino que estos beneficios se limitan solamente al plano familiar o individual. No ven que haya avances en el desarrollo de infraestructura como construcción de centros recreativos o mejora de las calles y caminos comunales que permitirían mejorar la calidad de vida en la comunidad en general. El principal cambio percibido es a nivel de infraestructura individual como aumento de casas residenciales y cabinas de hospedaje.

Respecto del desarrollo social, Castellón identificó que los participantes en este estudio coinciden en que ahora es mejor la educación y asocian esto al turismo, dado que se hicieron mejoras al colegio (técnico) pensando en capacitar a los jóvenes para trabajar en este sector. Además, reconocen avances en la enseñanza del inglés y la informática. Sin embargo, también hubo coincidencia en la percepción de que la mayor parte del desarrollo urbano significativo ha ocurrido en las comunidades de Playa Tamarindo o Playa Flamingo (no tanto en Villarreal ni en otras comunidades no colindantes directamente con la playa) y más que todo por inversión de capital extranjero. Pese a lo anteriormente citado, estos pobladores consideran que el Estado ha hecho inversiones en servicios públicos básicos (agua potable, electricidad, telecomunicaciones, salud y educación) que han mejorado la calidad de vida en la comunidad.

Castellón también indagó sobre el impacto del Turismo en la cultura de esta Comunidad de Villarreal, encontró que no existe tanta claridad sobre la existencia de cambios significativos en las costumbres del lugar que se sean atribuibles a esta actividad. Donde algunos sí observan cambios es en el segmento de los jóvenes, relacionados con su comportamiento o forma de vestir, atribuidos al contacto con turistas y migrantes ligados a la actividad turística. Blanco (2016, 238-240) señala aspectos negativos del impacto del turismo en la provincia, al indicar que ha ejercido presión sobre los recursos naturales; ha consumido bosques, agua y otros recursos naturales; además, contamina el ambiente con importantes cantidades de basura, lo cual afecta a los ecosistemas. La actividad turística ha tenido su Meca en las zonas costeras de Guanacaste, lo que ha ejercido presión sobre el recurso hídrico de las poblaciones locales, por el uso intensivo de este recurso. En 2007 algunas estimaciones calculan que operaban cerca de 90 pozos de agua clandestinos. En Tambor y Sámara se talaron bosques y drenaron manglares, así como también rellenaron esteros en otros lugares para abrir 
proyectos turísticos. Playa Flamingo se vio abrumada por cantidades de cloacas y botaderos de basura al aire libre. Otros impactos similares ocurrieron también en Tamarindo y Playa Grande.

Chen y García (2010) realizaron un estudio de percepción sobre el impacto del Turismo en el Roble 2 de Puntarenas y encontraron que, en general, prevalecía una percepción positiva sobre esta actividad, siendo esta percepción mayor entre quienes trabajaban en el sector Turismo y aquellos de menores ingresos económicos. Como efectos positivos, se señaló una mejoría en la calidad de vida, notándose aquí una opinión diferenciada (más negativa que positiva) de aquellas personas que laboraban para el Sector Público. Entre los aspectos negativos de esta actividad que encontraron estas investigadoras, estuvieron el maltrato a la naturaleza, la drogadicción y prostitución, y también que incide en el desplazamiento de costarricenses por extranjeros que adquieren tierras y están recibiendo mayor atenciones por parte de los proveedores de servicios estatales.

\section{Materiales y métodos}

El enfoque de la investigación es cuantitativo; es un diseño no experimental, de tipo descriptivo. La técnica de recolección de los datos empleada fue la entrevista "cara a cara" en el hogar de las personas entrevistadas. El instrumento utilizado fue un cuestionario.

El tamaño de muestra tuvo un $95 \%$ de confianza, con un error de 5.6\%, con corrección por finitud y 5\% de no respuesta de 644 personas; dicho cálculo fue contratado al Instituto Nacional de Estadística y Censos, y es representativa de toda la provincia de Guanacaste.

El tipo de muestreo empleado se hizo varias etapas, siendo la primera etapa la estratificación por cantón, seguido de un muestreo por conglomerados (o unidades primarias de muestreo, UPM) donde la selección de estas UPM fue por medio de un muestreo con probabilidad proporcional al tamaño del conglomerado (PPT). La selección final de las personas entrevistadas se hizo mediante una selección de cuota por edad y género, que garantiza una representatividad por estas dos características. Esta selección se realizó tomando en cuenta la distribución relativa de las personas de 18 y más años de Guanacaste, distribuidas por cantón, por grupo de edad y por género, según datos del Censo de Población y Vivienda del 2011. La distribución planeada de la muestra por cantón, se presenta en el cuadro 1, a continuación: 
Cuadro 1

Guanacaste: distribución de la muestra planeada

según cantón. Marzo, 2016

\begin{tabular}{ll}
\hline Cantón & $\begin{array}{l}\text { Número } \\
\text { personas }\end{array}$ \\
\hline Liberia & 130 \\
Nicoya & 100 \\
Santa Cruz & 110 \\
Bagaces & 40 \\
Carrillo & 70 \\
Cañas & 50 \\
Abangares & 40 \\
Tilarán & 40 \\
Nandayure & 20 \\
La Cruz & 40 \\
Hojancha & 10 \\
\hline Total & $\mathbf{6 5 0}$ \\
\hline
\end{tabular}

En total se eligieron 65 UPM y, en cada uno de estos conglomerados se seleccionaron 10 personas, para un total de 650 .

\section{Resultados y discusión}

De las 650 personas de la muestra meta, se logró entrevistar a 640 personas, lo cual no afecta la precisión del estudio dado que el tamaño de muestra había sido inflado un 5\%, con el fin de contemplar aquellos casos en donde no se lograra completar el tamaño de muestra requerido, para un 5,6\% de error planificado.

\section{Perfil de los entrevistados}

Se entrevistaron 316 hombres y 324 mujeres, en cifras relativas, 49,4\% y 50,6\%, respectivamente.

El cuadro 2 presenta la distribución por grupos de edad, en donde se observa que 3 personas no respondieron a esta pregunta. 


\section{Cuadro 2}

Guanacaste: distribución de una muestra de 640 personas mayores de 18 años según grupo de edad. Periodo Julio a Setiembre de 2018

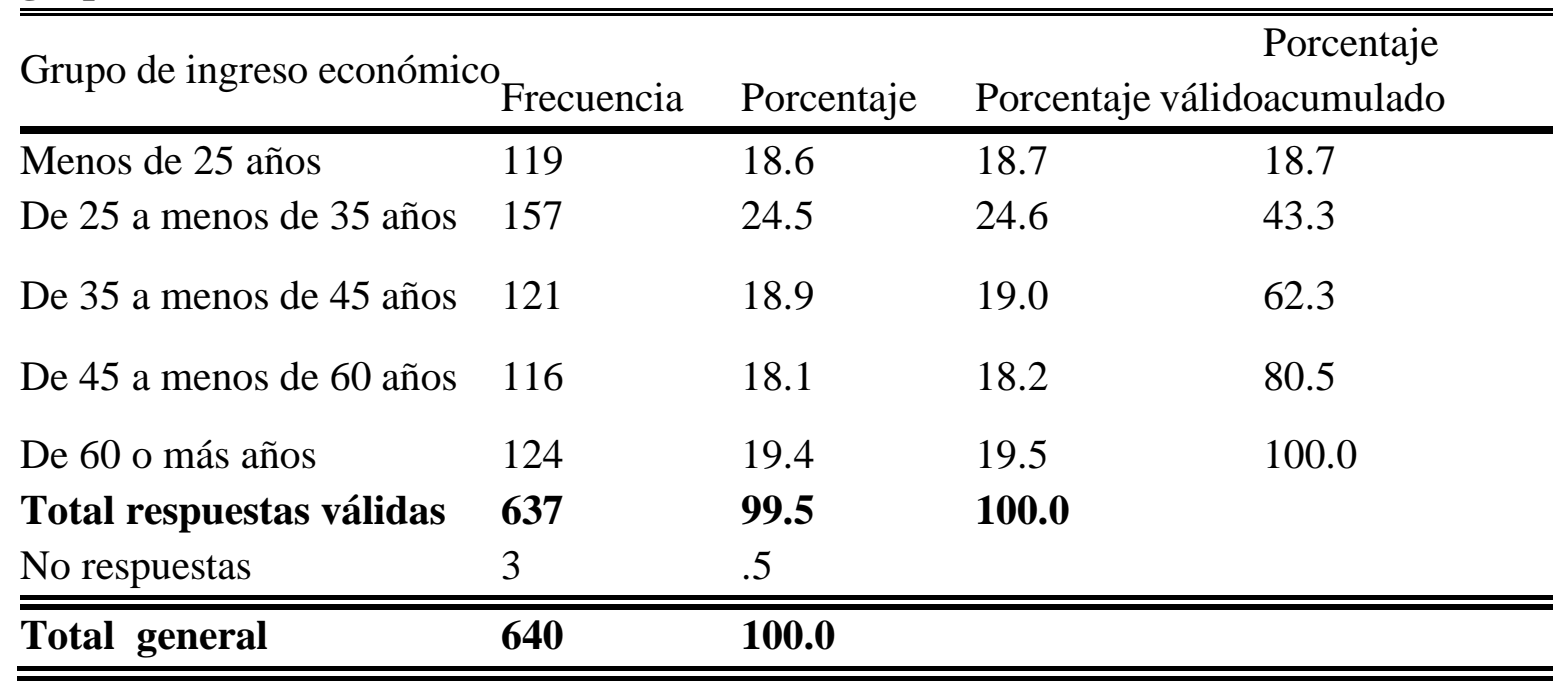

El cuadro 3 presenta los resultados agrupados según el estado civil, siendo las categorías más frecuentes soltero, casado y unión libre, en ese orden de frecuencia.

El cuadro 4 contiene los resultados por nivel de escolaridad, en donde se observa que aproximadamente 8 de cada 10 personas tienen un nivel máximo de secundaria completa. Mientras, el cuadro 5 contiene los datos agrupados por actividad económica, siendo la ocupación de servicios la más frecuente entre las personas activas económicamente hablando, y las amas de casa las más frecuentes dentro de las personas clasificadas como inactivas. En términos globales, incluyendo ambas actividades, las amas de casa fue el grupo de la población con mayor participación en este estudio. También se indagó si las personas entrevistadas eran nativas de la comunidad o procedían de otro lugar; el 68\% manifestó ser nativos, mientras un 32 procedente de otro lugar.

Cuadro 3

Guanacaste: distribución de una muestra de 640 personas mayores de 18 años según estado civil. Periodo Julio a Setiembre de 2018

\begin{tabular}{llll}
\hline \hline Estado civil & Frecuencia & Porcentaje & Porcentaje válido \\
\hline Soltero (a) & 206 & 32.2 & 32.4 \\
Casado(a) & 196 & 30.6 & 30.8 \\
Viudo(a) & 20 & 3.1 & 3.1 \\
Divorciado (a) & 49 & 7.7 & 7.7 \\
Unión libre & 165 & 25.8 & 25.9 \\
Total respuestas válidas & $\mathbf{6 3 6}$ & $\mathbf{9 9 . 4}$ & $\mathbf{1 0 0 . 0}$ \\
No respuestas & 4 & .6 & \\
\hline \hline Total General & $\mathbf{6 4 0}$ & $\mathbf{1 0 0 . 0}$ & \\
\hline \hline
\end{tabular}




\section{Cuadro 4}

Guanacaste: distribución de una muestra de 640 personas mayores de 18 años según nivel de escolaridad. Periodo Julio a Setiembre de 2018

\begin{tabular}{lllll}
\hline \hline Nivel de escolaridad & Frecuencia & Porcentaje & Porcentaje válido acumulado \\
\hline Ninguna & 15 & 2.3 & 2.4 & 2.4 \\
Primaria incompleta & 84 & 13.1 & 13.2 & 15.5 \\
Primaria completa & 146 & 22.8 & 22.9 & 38.4 \\
Secundaria incompleta & 161 & 25.2 & 25.2 & 63.6 \\
& & & & 78.4 \\
Secundaria completa & 94 & 14.7 & 14.7 & 87.9 \\
Superior incompleta & 61 & 9.5 & 9.6 & 100.0 \\
Superior completa & 77 & 12.0 & 12.1 & \\
Total respuestas válidas & $\mathbf{6 3 8}$ & $\mathbf{9 9 . 7}$ & $\mathbf{1 0 0 . 0}$ & \\
No respuestas & 2 & .3 & & \\
\hline \hline Total & $\mathbf{6 4 0}$ & $\mathbf{1 0 0 . 0}$ \\
\hline \hline
\end{tabular}

\section{Cuadro 5}

Guanacaste: distribución de una muestra de 640 personas mayores de 18 años según actividad económica a la que dedica la mayor parte de su tiempo.

Periodo julio a setiembre, 2018

\begin{tabular}{|c|c|c|c|}
\hline & Frecuencia & Porcentaje & $\begin{array}{l}\text { Porcentaje } \\
\text { válido }\end{array}$ \\
\hline \multicolumn{3}{|l|}{ ACTIVOS } & \\
\hline $\begin{array}{l}\text { Ocupaciones de venta en locales y de prestación de } \\
\text { servicios directos a personas }\end{array}$ & 67 & 22,3 & 12.0 \\
\hline $\begin{array}{l}\text { Ocupaciones calificadas agropecuarias, agrícolas y } \\
\text { pesqueras }\end{array}$ & 56 & 18,7 & 10.0 \\
\hline $\begin{array}{l}\text { Producción artesanal, la construcción, la mecánica y } \\
\text { artes mecánicas, artes gráficas y la industria } \\
\text { manufacturera }\end{array}$ & 51 & 17,0 & 9.1 \\
\hline Ocupaciones no calificadas & 39 & 13,0 & 7.0 \\
\hline Ocupaciones de nivel técnico y profesional medio & 29 & 9,7 & 5.2 \\
\hline $\begin{array}{l}\text { Ocupaciones de nivel profesional, científico e } \\
\text { intelectual }\end{array}$ & 23 & 7,7 & 4.1 \\
\hline Ocupaciones de apoyo administrativo & 18 & 6,0 & 3.2 \\
\hline $\begin{array}{l}\text { Ocupaciones de montaje y de operación de } \\
\text { instalaciones y de máquinas }\end{array}$ & 15 & 5,0 & 2.7 \\
\hline
\end{tabular}




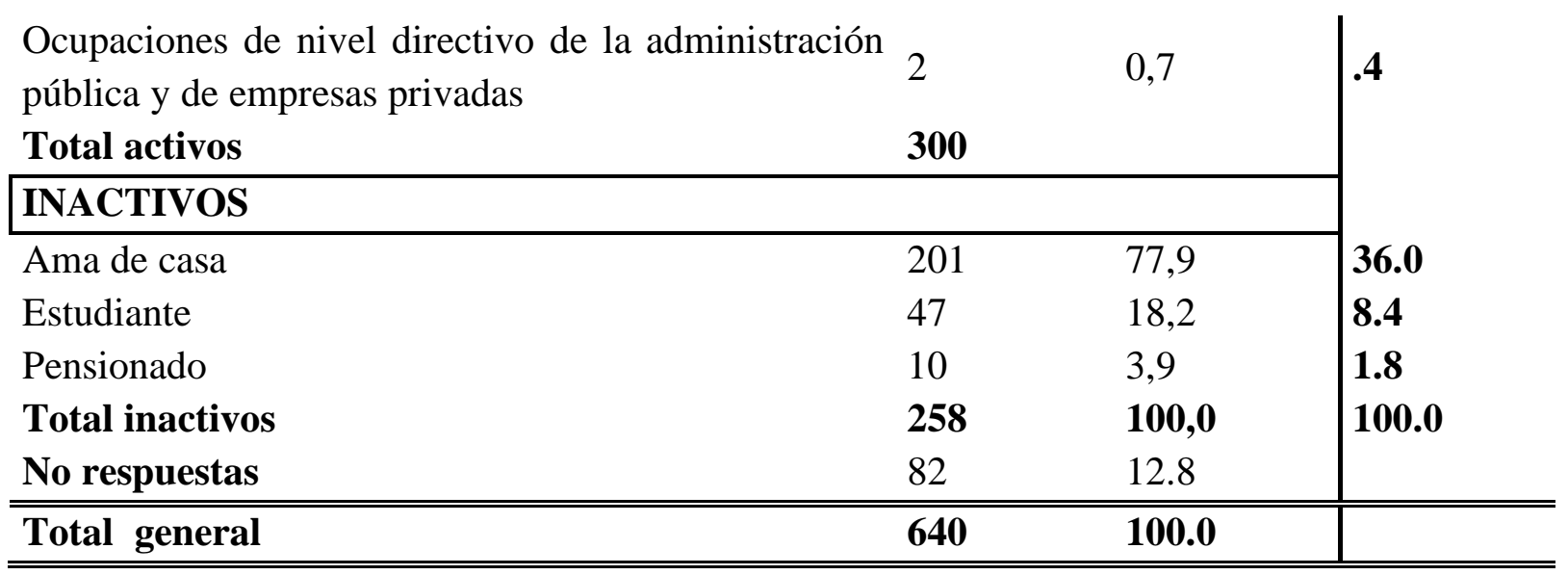

\section{Resultados sobre la percepción respecto del turismo}

Un día, hace unos 5 años, se acercó ejecutiva de la Cámara de Turismo de Guanacaste a la Sede Guanacaste de la Universidad de Costa Rica, interesada en que se pudiera hacer una investigación para determinar qué opinión tenían los guanacastecos respecto del Turismo. Esta investigación nace como resultado del interés de esta Cámara y del investigador principal, en quien ese interés quedó latente; hasta ese momento esta pregunta no había tenido respuesta; mucho se hablaba del impacto del turismo, pero no se había consultado a los residentes en la provincia su opinión al respecto. Hoy, finalmente esa pregunta tiene respuesta, y estos resultados se presentan a continuación.

La pregunta central de este estudio versó sobre si el Turismo había traído solo beneficios a la comunidad, más beneficios que problemas, beneficios y problemas en la misma proporción, más problemas que beneficios o solo problemas. Esas fuerón las opciones de respuesta dadas a los entrevistados. De las 640 personas consultadas, se clasificó a 143 como "no aplicables" para esta pregunta, dado que vivían en lugares de poco o ningún proyecto o lugar turístico $(22,3 \%)$ y 4 personas no respondieron $(0.6 \%)$. Las respuestas válidas correspondieron a $493(77 \%)$ Los resultados que se presentan en el gráfico 1 corresponden a este grupo de respuestas válidas.

De este gráfico se desprende que 3 de cada 4 personas ven más positivo el turismo que negativo y, aproximadamente, 2 de cada 5 personas lo ven solo como positivo, esto es, que trae solo beneficios. Solo 1 de cada 20 personas considera que el Turismo trae más problemas que beneficios, o bien, solo trae problemas. 


\section{Gráfico 1}

Guanacaste: distribución de una muestra de 493 personas residentes en la provincia según su percepción sobre el impacto del turismo en la comunidad. Julio a setiembre, 2018

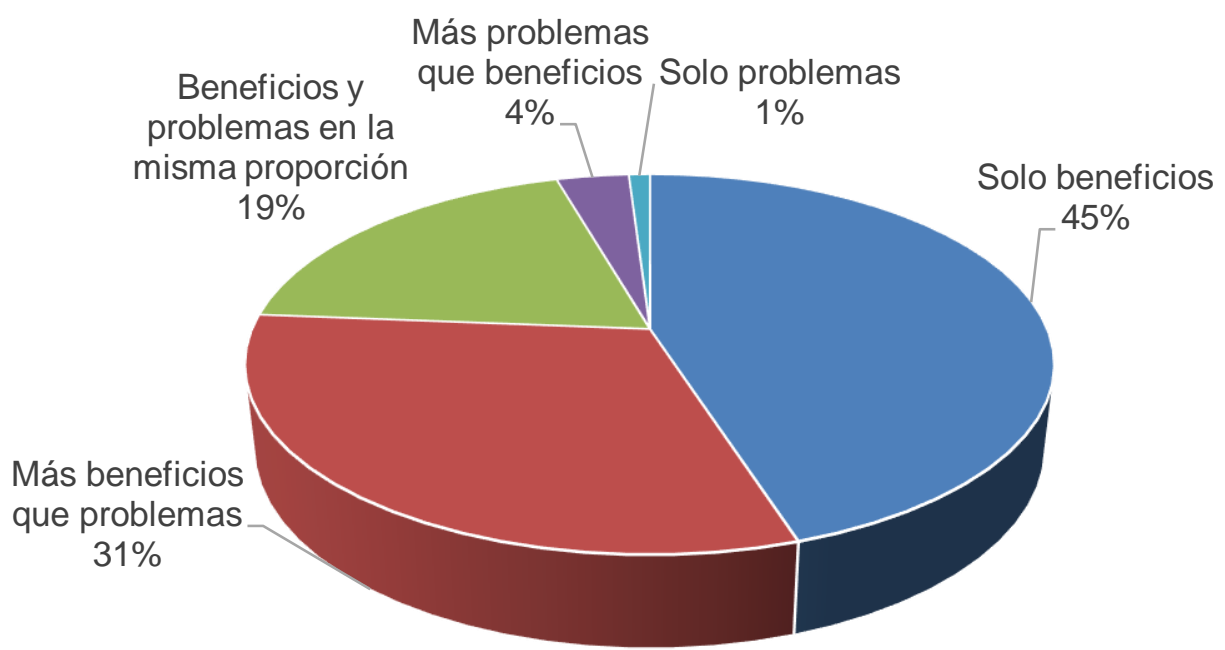

El cuadro 6 presenta los beneficios que los entrevistados le atribuyen al Turismo, destacando las fuentes de trabajo y una fuente de ingresos económicos. En este cuadro se presentan dos tipos de números relativos; el primero, basado en el total de respuestas, y el segundo, en el total de personas que respondieron. Cuarenta y cuatro de cada cien personas consideran que el turismo trae consigo fuentes de trabajo.

El cuadro 7 presenta los resultados sobre los problemas que atribuyen los entrevistados a la actividad turística en su comunidad; se observa ahí que la mitad de los entrevistados considera que esa actividad no produce ningún problema, siendo esa la opinión más frecuente. Dentro de los que sí manifestaron la existencia de problemas (basado en la columna porcentaje de casos) la drogadicción, y el alcoholismo y la falta de agua fueron citados como los dos principales, seguidos un poco más de lejos por la contaminación.

El cuadro 8 presenta los datos sobre el cruce de variables entre las variables opinión sobre los beneficios del turismo y si trabaja o no en ese sector, se observa ahí que la opinión sobre el impacto o los beneficios del Turismo es mayor cuando no se trabaja en ese sector, que cuando se trabaja ahí. Una prueba Chi Cuadrado (valor $\mathrm{p}=0,004$ ) indicó que ambas variables estaban relacionadas, esto es, que la opinión sobre el nivel de beneficios o los problemas del Turismo en la comunidad depende del hecho de trabajar o no en esa actividad económica. Además, se hicieron pruebas Chi Cuadrado para el el cruce de esta variable, opinión sobre los beneficios y problemas del Turismo, con las variables edad (valor $p=0,042$ ), nivel de escolaridad (valor $\mathrm{p}=0,229$ ) e ingreso económico (valor $\mathrm{p}=0,801$ ). También se corrieron correlaciones Spearman para el cruce de las variables citadas, con el fin de medir el nivel de correlación, pero ninguna resultó significativa (solo una, la correlación entre nivel de escolaridad y el nivel de beneficios o problemas, tuvo un valor $\mathrm{p}$ de $0 ., 030$, significativo a un 
nivel alfa de 0,05, pero con un valor muy bajo para el coeficiente, 0,098) ; esto implicó, que la prueba no demostró relación entre las variables ya mencionadas.

\section{Cuadro 6}

Guanacaste: distribución de una muestra de 640 personas residentes en la provincia según su opinión sobre los beneficios que le señalan al Turismo en su comunidad. Julio a setiembre, 2018

\begin{tabular}{llll}
\hline \multirow{2}{*}{$\begin{array}{l}\text { Beneficios del Turismo } \\
\text { Fuentes de trabajo }\end{array}$} & Cantidad & Porcentaje & $\begin{array}{l}\text { Porcentaje de } \\
\text { casos }\end{array}$ \\
\cline { 2 - 4 } $\begin{array}{l}\text { Mayor dinamismo } \\
\text { económicos }\end{array}$ & 284 & 27,8 & 44,4 \\
Mayor reconocimiento y mejoras en & 97 & 21,2 & 33,9 \\
la comunidad & & & \\
Mejoras a la educación & 83 & 9,5 & 15,2 \\
Distracción, recreación & 39 & 8,1 & \\
Intercambios culturales & 34 & 3,8 & 6,1 \\
Mejoras en la salud y bienestar social & 29 & 3,3 & 5,3 \\
Aprendizaje de idiomas & 24 & 2,8 & 4,5 \\
Conservación de medio ambiente & 9 & 2,3 & 3,8 \\
Ningun beneficio & 8 &, 9 & 1,4 \\
No sabe, no responde, no aplica & 198 &, 8 & 1,3 \\
\hline Total & $\mathbf{1 0 2 2}$ & $\mathbf{1 0 0 , 0 0 0}$ & \\
\hline
\end{tabular}

\section{Cuadro 7}

Guanacaste: distribución de una muestra de 640 personas residentes en la provincia según su opinión sobre los problemas que le señalan al Turismo en su comunidad. Julio a setiembre, 2018

\begin{tabular}{llll}
\hline \hline \multirow{2}{*}{ Problemas en la comunidad } & Respuestas & & $\begin{array}{l}\text { Porcentaje } \\
\text { Ninguno }\end{array}$ \\
\cline { 2 - 3 } & Cantidad & Porcentaje & casos \\
\hline Drogadicción, alcoholismo & 322 & 41,1 & 50,3 \\
Falta de agua & 97 & 12,4 & 15,2 \\
Contaminación & 77 & 9,8 & 12,0 \\
Explotación de recursos naturales & 36 & 4,6 & 5,6 \\
Delincuencia & 31 & 4,5 & 5,5 \\
Daño a medio ambiente & 19 & 4,0 & 4,8 \\
Pérdida de valores & 17 & 2,4 & 3,0 \\
& & 2,2 & 2,7
\end{tabular}




\begin{tabular}{|c|c|c|c|}
\hline Abusos en zonas protegidas & 13 & 1,7 & 2,0 \\
\hline Altos precios & 13 & 1,7 & 2,0 \\
\hline Explotación del personal & 10 & 1,3 & 1,6 \\
\hline Problemas con vías de acceso & 9 & 1,1 & 1,4 \\
\hline Daño al medio ambiente & 9 & 1,1 & 1,4 \\
\hline Transculturación & 8 & 1,0 & 1,3 \\
\hline Llegada de extranjeros & 6 &, 8 & ,9 \\
\hline $\begin{array}{l}\text { Atracción de capital de dudosa } \\
\text { procedencia }\end{array}$ & 5 & ,6 &, 8 \\
\hline Inseguridad & 5 & ,6 & ,8 \\
\hline Aumento de impuestos & 3 & ,4 &, 5 \\
\hline Lavado de dólares y otros similares & 2 & ,3 &, 3 \\
\hline Conflictos & 2 & ,3 &, 3 \\
\hline Daño a infraestructura & 2 &, 3 &, 3 \\
\hline Ingresos económicos para pocos & & & \\
\hline pobladores & 1 &, 1 & ,2 \\
\hline Falta de trabajo & 1 &, 1 & ,2 \\
\hline Indigentes & 1 &, 1 &, 2 \\
\hline Falta de interés en los jóvenes & 1 &, 1 & ,2 \\
\hline Aumento de desigualdad & 1 &, 1 & ,2 \\
\hline Prostitución & 1 &, 1 & ,2 \\
\hline Privatización & 1 &, 1 &, 2 \\
\hline Consecuencias a la ganadería & 1 &, 1 & ,2 \\
\hline No sabe, no responde & 55 & 7,0 & 8,6 \\
\hline Total & 784 & 100,0 & \\
\hline
\end{tabular}

Se hizo una prueba de dependencia bajo Chi Cuadrado para determinar la dependencia de la variable central de estudio, con el estado civil (agrupado en soltero(a), casado o en unión libre, y divorciado(a) o viudo(a)); el resultado arrojó un valor p igual a 0,196 que estas variables no están relacionadas.

Con respecto al género, la prueba Chi Cuadrado arrojó un valor p igual a 0,273, la cual señala que existe independencia entre la opinión sobre los beneficios o problemas que trae el Turismo a la comunidad y si se es hombre o mujer. Se adjunta el cuadro 9 con los resultados del cruce entre estas variables. Aunque se observaron, algunas diferencias por género, en la percepción, la prueba no encontró diferencias estadísticamente significativas para afirmar que la percepción sobre el impacto del Turismo tiene alguna relación con el género. 


\section{Cuadro 8}

Guanacaste: distribución de una muestra de 493 personas residentes en la provincia según su percepción sobre el impacto del turismo

en la comunidad y si trabaja o no en ese sector.

Julio a setiembre, 2018

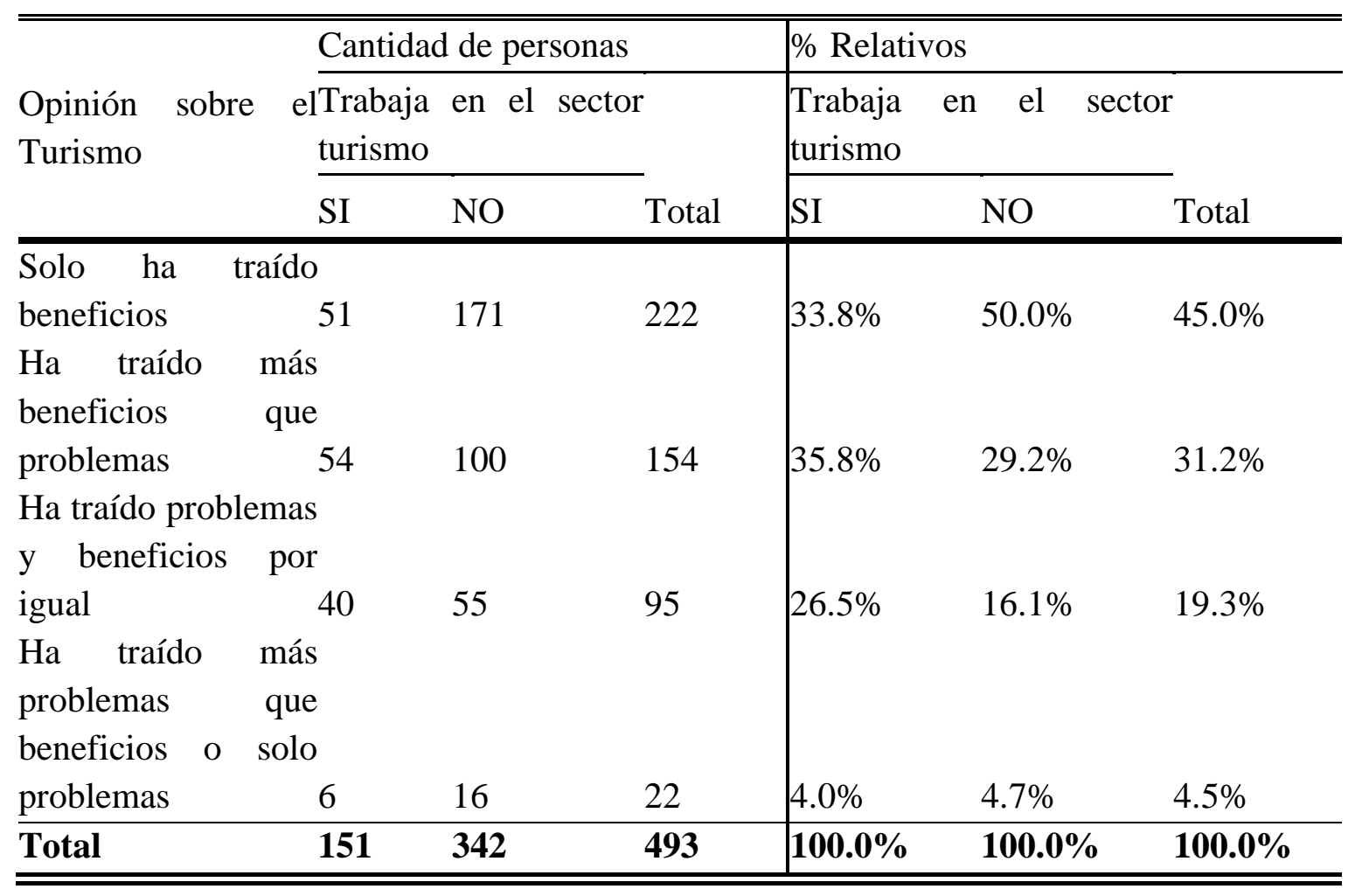




\section{Cuadro 9}

Guanacaste: distribución de una muestra de 493 personas residentes en la provincia según su percepción sobre el impacto del turismo en la comunidad por género. Julio a setiembre, 2018

(Valores relativos)

\begin{tabular}{|c|c|c|c|}
\hline \multirow{2}{*}{ Opinión sobre el Turismo } & \multicolumn{2}{|l|}{ Genero } & \multirow[b]{2}{*}{ Total } \\
\hline & Femenino & Masculino & \\
\hline Total de entrevistados & 238 & 255 & 493 \\
\hline Total & $100,0 \%$ & $100,0 \%$ & $100,0 \%$ \\
\hline $\begin{array}{l}\text { El turismo solo ha traído beneficio } \\
\text { a la comunidad }\end{array}$ & $\mathrm{s} 42.9 \%$ & $47.1 \%$ & $45.0 \%$ \\
\hline $\begin{array}{l}\text { El turismo ha traído problemas } \\
\text { beneficios en la misma cantidad }\end{array}$ & $\mathrm{y} 22.3 \%$ & $16.5 \%$ & $19.3 \%$ \\
\hline $\begin{array}{l}\text { El turismo ha traído más problema } \\
\text { que beneficios a la comunidad } \\
\text { solo problemas }\end{array}$ & s $3.4 \%$ & $5.5 \%$ & $4.5 \%$ \\
\hline$\overline{\text { Total }}$ & $100.0 \%$ & $100.0 \%$ & $100.0 \%$ \\
\hline
\end{tabular}

También se consultó a las personas que, si eran nativos de la comunidad o provenían de otro lugar, y esta variable se cruzó con la variable central de esta investigación, se hizo la prueba Chi Cuadrada como en los otros casos anteriores, y se encontró un valor p igual a 0,345. El cuadro 10 presenta estos datos, de forma similar al cuadro anterior; aunque se observan algunas diferencias en el nivel de percepción, estas no resultaron estadísticamente significativas, por lo tanto, no se puede afirmar que la percepción sobre el impacto del Turismo en la comunidad dependa de si la persona es nativa o no de la zona. 


\section{Cuadro 10}

Guanacaste: distribución de una muestra de 481 personas residentes en la provincia según su percepción sobre el impacto del turismo en la comunidad y si es o no nativo de la comunidad.

Julio a setiembre, 2018

(Valores relativos)

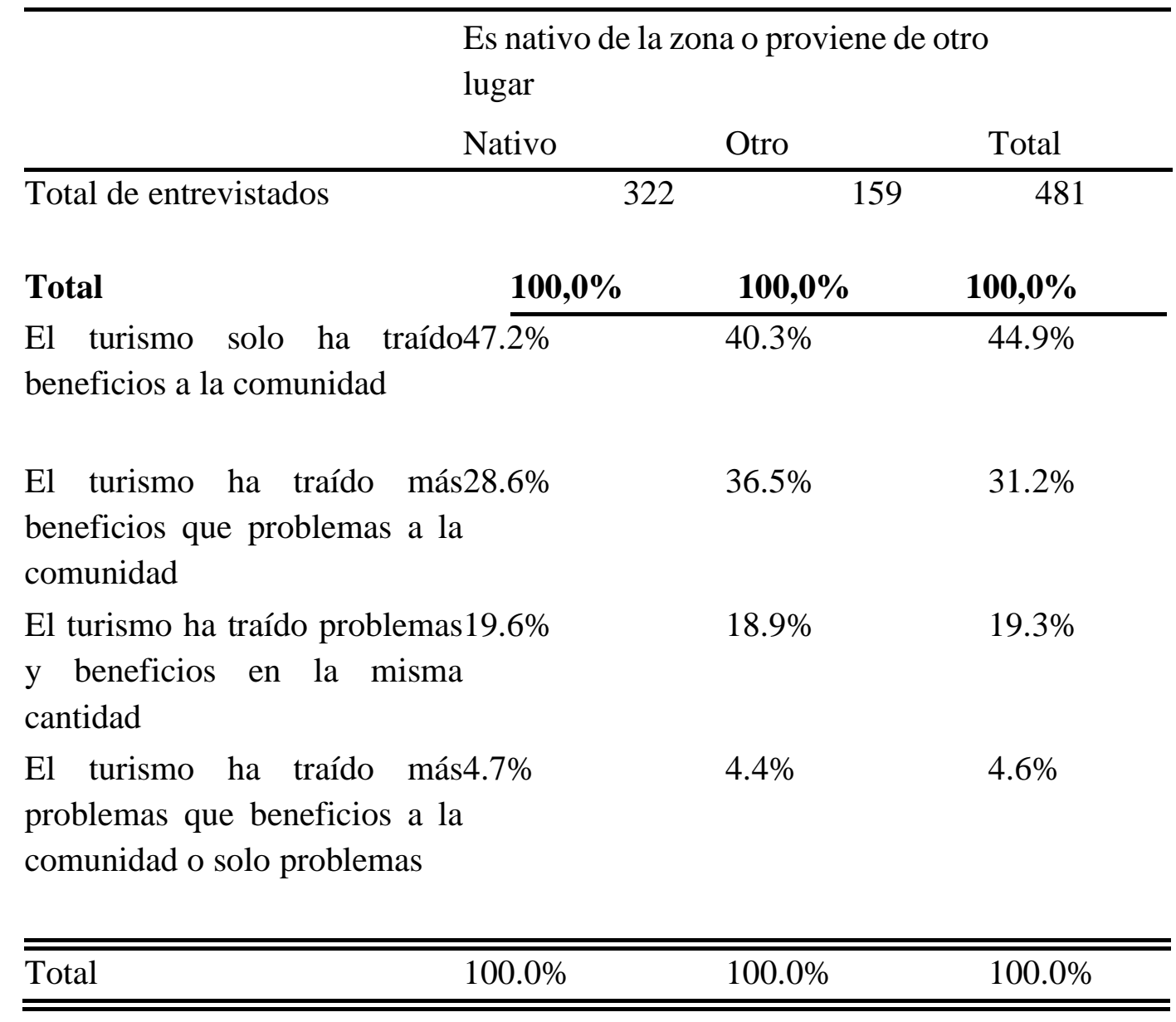

El cuadro 11 presenta la opinión sobre el impacto del Turismo clasificado por cantón; aquellos cantones con pocos datos y distribuciones relativas similares se agruparon para dar mayor consistencia a los datos; solo se hizo una excepción con el cantón de Nandayure, cuyo comportamiento relativo era muy diferente de otros cantones "pequeños" de la provincia. Por esta razón se decidió no agruparlo, aunque la cantidad pequeña de entrevistados le reste confiabilidad a los datos. Interesante resulta, de lo presentado en este cuadro, que dos cantones con quizá la mayor visitación de Turismo de Playa, presentaron opiniones menos favorables hacia los beneficios del turismo que las expresadas por otros cantones con mucho menos visitación de turistas. Esto corresponde a los cantones de Santa Cruz y Carrillo, los cuales cuentan con importantes centros de actividad turística en las Playas de Tamarindo, Carrillo y el Polo Turístico de la Península de Papagayo. 


\section{Cuadro 11}

Guanacaste: distribución de una muestra de 481 personas residentes en la provincia según su percepción sobre el impacto del turismo

en la comunidad y por cantòn.

Julio a setiembre, 2018

(Valores relativos)

\begin{tabular}{|c|c|c|c|c|c|c|}
\hline \multirow[b]{2}{*}{ Cantón } & \multirow[b]{2}{*}{$\begin{array}{l}\text { Cantidad } \\
\text { de } \\
\text { personas }\end{array}$} & \multicolumn{5}{|c|}{ Opinión sobre impacto del Turismo \% } \\
\hline & & $\begin{array}{l}\text { Tota } \\
1\end{array}$ & $\begin{array}{l}\text { Solo trae } \\
\text { beneficios }\end{array}$ & $\begin{array}{l}\text { Más } \\
\text { beneficios } \\
\text { que } \\
\text { problemas }\end{array}$ & $\begin{array}{l}\text { Beneficios } \\
\mathbf{y} \\
\text { problemas } \\
\text { por igual }\end{array}$ & $\begin{array}{l}\text { Más } \\
\text { problemas } \\
\text { que } \\
\text { beneficios o } \\
\text { solo } \\
\text { problemas }\end{array}$ \\
\hline Liberia & 93 & 100,0 & 51,6 & 26,9 & 17,2 & 4,3 \\
\hline Nicoya & 74 & 100,0 & 47,3 & 37,8 & 12,2 & 2,7 \\
\hline Santa & 94 & 100,0 & & & & \\
\hline Cruz & & & 23,4 & 34,0 & 35,1 & 7,4 \\
\hline Bagaces- & 60 & 100,0 & & & & \\
\hline Cañas & & & 61,7 & 28,3 & 8,3 & 1,7 \\
\hline Carrillo & 68 & 100,0 & 33,8 & 33,8 & 29,4 & 2,9 \\
\hline Abangares & 48 & 100,0 & & & & \\
\hline - Tilarán & & & 54,2 & 37,5 & 2,1 & 6,3 \\
\hline Nandayur & 19 & 100,0 & & & & \\
\hline & & & 26,3 & 36,8 & 21,1 & 15,8 \\
\hline $\begin{array}{l}\text { La Cruz- } \\
\text { Hojancha }\end{array}$ & 37 & 100,0 & 70,3 & 10,8 & 18,9 & 0,0 \\
\hline $\begin{array}{l}\text { Total } \\
\end{array}$ & 493 & 100,0 & $\overline{45,0}$ & 31,2 & 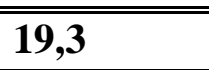 & $\overline{4,5}$ \\
\hline
\end{tabular}

El gráfico 2 muestra el perfil que tienen las personas residentes en la provincia sobre el Turismo; los tres principales adjetivos con que calificaron esta actividad económica fueron:

- Más beneficioso que perjudicial.

- Más aburrido que divertido.

- Más ecológico que de otra índole.

En resumen, beneficioso, divertido y ecológico fueron los tres principales adjetivos con que las personas, residentes en Guanacaste, calificaron al Turismo. En contraposición, cultural, extranjero y de playa fueron los tres calificativos con los que menos identificaron al Turismo en la región. Estos términos se obtuvieron a través de la aplicación de un diferencial semántico, en donde valoraron adjetivos opuestos en una escala de -3 a 3 , siendo -3 el valor más alto para los adjetivos a la izquierda y 3 , el más alto para los adjetivos de la derecha, de 
cada par que se presentan en el eje de categorías del gráfico. Si un adjetivo hubiese tenido un puntaje negativo, entonces el adjetivo de la izquierda es el que se hubiera usado para identificar al Turismo. En este caso, solo resultaron puntajes promedio positivos; por lo tanto, para determinar el perfil de esta actividad, se eligieron los adjetivos a la derecha. Por ejemplo, en el caso de "Montaña-Playa", el puntaje medio fue positivo, lo cual indicó eso un turismo más de playa que de montaña, aunque su bajo puntaje señaló que existe cierta indecisión al respecto en los pobladores, igual análisis aplicaría para "Nacional-Extranjero".

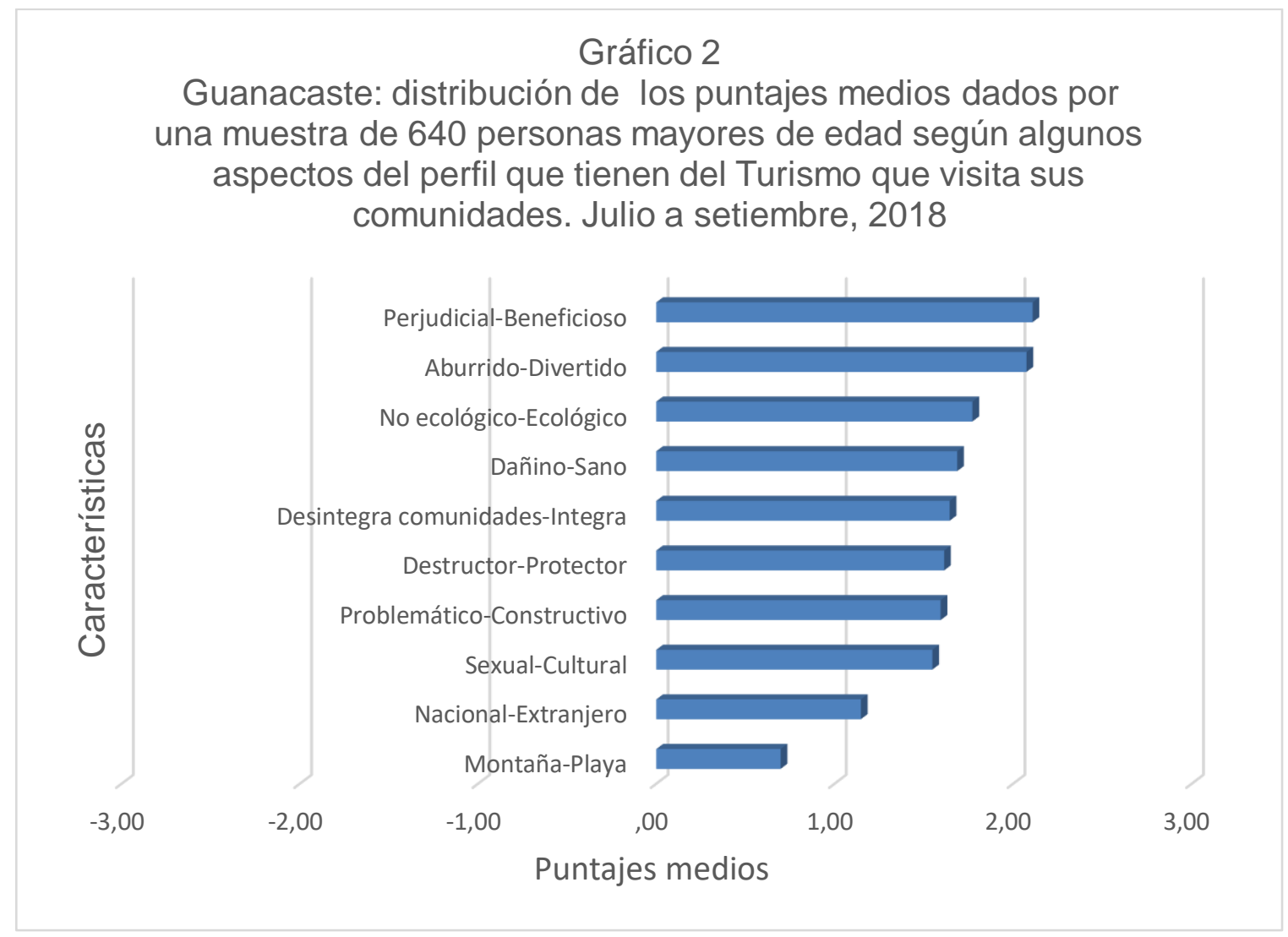

La tabla 1 siguiente, presenta los adjetivos con los que los guanacastecos identificaron al Turismo, ordenados de mayor a menor mención. Se observa ahí que, en general, existe una alta variabilidad (coeficiente de variación) en las opiniones respecto de cada uno de los adjetivos del perfil que tienen las personas de la provincia sobre el Turismo. Y también ser observa que, cuanto menor es el puntaje asignado a cada uno de estos términos, mayor tiende a ser la variabilidad respecto a ese adjetivo. Esto indica que los calificativos con mayor mención, además, son aquellos en los que la opinión de los pobladores de la provincia es más consistente; quiere decir que, existe menos duda.

\section{Tabla 1}

\section{Perfil del Turismo}




\begin{tabular}{|c|c|c|c|c|c|c|}
\hline & $\begin{array}{l}\text { Cantidad } \\
\text { de } \\
\text { personas }\end{array}$ & Mínimo & Máximo & Media & $\begin{array}{l}\text { Desviación } \\
\text { estándar }\end{array}$ & $\begin{array}{l}\text { Coeficiente } \\
\text { de } \\
\text { variación }\end{array}$ \\
\hline Beneficioso & 620 & -3 & 3 & 2,11 & 1,248 & $59 \%$ \\
\hline Divertido & 615 & -3 & 3 & 2,08 & 1,465 & $71 \%$ \\
\hline Ecológico & 616 & -3 & 3 & 1,77 & 1,541 & $87 \%$ \\
\hline Sano & 617 & -3 & 3 & 1,69 & 1,473 & $87 \%$ \\
\hline Integrador & 617 & -3 & 3 & 1,65 & 1,541 & $94 \%$ \\
\hline Protector & 622 & -3 & 3 & 1,61 & 1,615 & $100 \%$ \\
\hline Constructivo & 621 & -3 & 3 & 1,59 & 1,632 & $102 \%$ \\
\hline Cultural & 611 & -3 & 3 & 1,55 & 1,504 & $97 \%$ \\
\hline Extranjero & 621 & -3 & 3 & 1,15 & 1,942 & $169 \%$ \\
\hline Playa & 626 & -3 & 3 &, 70 & 2,107 & $302 \%$ \\
\hline
\end{tabular}

\section{Conclusiones y comentarios}

Con relación a la cuestionante sobre qué pensaban los residentes en Guanacaste sobre el impacto del Turismo en la provincia era una pregunta sin resolver; muchas especulaciones se hicieron al respecto, pero esta es la primera vez que un estudio de percepción sobre el impacto de esta actividad se lleva a cabo.

El estudio se hizo bajo un enfoque cuantitativo con muestra representativa y, por tanto, la opinión expresada tiene carácter de generalidad para las opiniones de los habitantes de toda la provincia. En general, la opinión que dan los y las residentes en la provincia al turismo son, por mucho, más positivas que negativas. Los beneficios mencionados están ligados a la generación de fuentes de trabajo e ingreso de divisas mientras en los problemas, "ninguno" fue la opinión más frecuentemente expresada. Entre los que mencionaron problemas, la drogadicción y el alcoholismo fueron los que tuvieron mayor presencia, coincidiendo esto con los resultados encontrados en el estudio de Chen y García llevado a cabo en el Roble de Puntarenas. Sin embargo, a diferencia de ese estudio del Roble de Puntarenas, en este sobre el Turismo en Guanacaste se encontró que un mayor ligamen (ocupaciones) a la actividad turística tendía a generar opiniones más negativas o menos positivas, que aquellos que no laboraban en este sector. Aparte de esta variable (trabajar en sector turismo), también se encontró asociación respecto de la edad de los entrevistados, las personas más jóvenes tienden a tener una opinión más favorable sobre los beneficios del Turismo; a medida que la edad aumenta, esta opinión va disminuyendo.

En esta investigación no se encontraron asociaciones entre la percepción sobre el Turismo y las variables sociodemográficas como género, estado civil, escolaridad e ingreso económico. También se analizó si existía dependencia entre la opinión sobre si el turismo traía beneficios 
o problemas a la comunidad y la condición de ser nativo o proceder de otro lugar; aquí tampoco se encontró asociación entre estas variables.

Beneficio, divertido y ecológico son los tres adjetivos con que identificaron mejor los residentes en Guanacaste al Turismo.

Otra conclusión importante es que los cantones con mayor visitación de Turismo, sobre todo de Playa (Santa Cruz y Carrillo), presentaron una percepción menos positiva respecto de los beneficios de esta actividad, en comparación con aquellos con menor exposición a este tipo de Turismo.

Aunque el estudio demostró que en general existe una opinión positiva respecto del al impacto del Turismo de Playa (o de visitación extranjera), también demostró que, a medida que hay mayor exposición a esta actividad (comunidades con mayor visitación y personas ocupadas en este sector), la percepción sobre los beneficios derivados disminuye. Por lo tanto, se recomienda investigar más sobre cuáles podrían ser las causas de esto, así como qué medidas tomarán, tanto las municipalidades como el Gobierno Central a la hora de decidir sobre más inversiones en este sector y la magnitud del canon cobrado por el desarrollo de esta actividad.

\section{Referencias bibliográficas}

Arrieta, G.y Rivera, G.. (2009). El desarrollo del Turismo en Guanacaste: De la Asociación Bella Vista, al Instituto Costarricense de Turismo en Historia de la (Re) Construcción de una región.1850-2007. Editorial Alma Mater, San José, Costa Rica. Págs. 139-156.

Blanco Obando, Eduardo. (2016). Naturaleza y producción: Efectos ambientales de las actividades productivas y la legislación ambiental en la región Chorotega de Costa Rica. Un análisis desde el metabolismo social, 1950-2014 en Guanacaste: Región e Historia 17852015. Editorial Alma Mater, San José, Costa Rica. Págs. 215-248.

Castellón, Luis A.. (2005). Percepción sobre el impacto del turismo en los valores sociales y la vida comunal de un grupo de pobladores de la Comunidad de Villarreal de Santa Cruz, Guanacaste. Tesis de grado de Licenciatura de la carrera de Psicología. Universidad de Costa Rica, Sede Guanacaste. Liberia, Guanacaste, Costa Rica.

Córdoba, C., Loría, D. y Picón, J.. (2011). Turismo en Guanacaste: del Modelo Tradicional al Modelo Sostenible. El caso de la comunidad de Nosara, Nicoya en (Re) Lecturas de Guanacaste 1821-2010. Sociedad Editora Alquimia 2000, S.A., San José, Costa Rica. Págs. 193-2016.

Chen, S., García, K. (2010). Percepción del impacto del Turismo en el Roble 2 de Puntarenas, Costa Rica. Rev. Reflexiones 89 (2): 27-38. 
Dirección General de Estadística y Censos. Censo Agropecuario de 1973. San José, Costa Rica.

Franco, E. y Sojo, C. (1992). Gobierno, empresarios y políticas de ajuste. Facultad Latinoamericana de Ciencias Sociales. San José, Costa Rica.

Gaete, Marcelo. (1990). ¿Qué es el ajuste estructural? Centro de Estudios y Publicaciones Alforja. San José, Costa Rica.

Loiaciga, M. (1994). Liberia: Desarrollo económico, social y cultural 1800 - 1980. Liberia, Guanacaste, Costa Rica. Universidad de Costa Rica, Sede Guanacaste. Liberia, Costa Rica. Volumen II. Págs. 22-44.

Sequeira, Wilder. (2009). "LAS ESTRUCTURAS AGRARIAS REGIONALES: Los pequeños y medianos productores agrícola - ganaderos de la Península de Nicoya (18501930) en Historia de la (Re) Construcción de una región.1850-2007. Editorial, Alma Mater. San José, Costa Rica. Págs. 55-77.

Solano, Edgar (2009). "Liberia, Ciudad del Cruce de caminos” en Guanacaste: Historia de la (Re)Construcción de una región 1850-2077. Editorial, Alma Mater. San José, Costa Rica. Págs. 81-100. 